\title{
Impact of Tumor Regional Involvement on Active Surveillance Outcomes: Validation of the Cumulative Cancer Location Metric in a US Population.
}

\author{
Joon Yau Leong \\ Thomas Jefferson University \\ Courtney Capella \\ Thomas Jefferson University \\ Seth Teplitsky \\ Thomas Jefferson University \\ Leonard G. Gomella

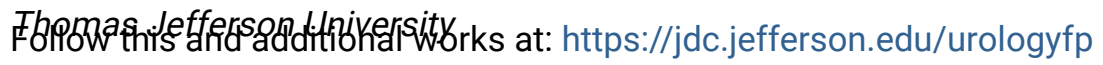 \\ orearcoffthterabutsibgy Commons, and the Urology Commons \\ Theras is feferso Uwiversitw access to this document benefits you
}

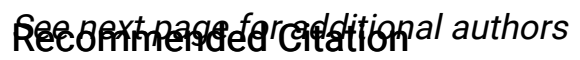

Leong, Joon Yau; Capella, Courtney; Teplitsky, Seth; Gomella, Leonard G.; Trabulsi, Edouard J.; Lallas, Costas D.; and Chandrasekar, Thenappan, "Impact of Tumor Regional Involvement on Active Surveillance Outcomes: Validation of the Cumulative Cancer Location Metric in a US Population." (2019). Department of Urology Faculty Papers. Paper 51.

https://jdc.jefferson.edu/urologyfp/51

This Article is brought to you for free and open access by the Jefferson Digital Commons. The Jefferson Digital Commons is a service of Thomas Jefferson University's Center for Teaching and Learning (CTL). The Commons is a showcase for Jefferson books and journals, peer-reviewed scholarly publications, unique historical collections from the University archives, and teaching tools. The Jefferson Digital Commons allows researchers and interested readers anywhere in the world to learn about and keep up to date with Jefferson scholarship. This article has been accepted for inclusion in Department of Urology Faculty Papers by an authorized administrator of the Jefferson Digital Commons. For more information, please contact: JeffersonDigitalCommons@jefferson.edu. 


\section{Authors}

Joon Yau Leong, Courtney Capella, Seth Teplitsky, Leonard G. Gomella, Edouard J. Trabulsi, Costas D. Lallas, and Thenappan Chandrasekar 
Title: Impact of Tumor Regional Involvement on Active Surveillance Outcomes: Validation of the Cumulative Cancer Location Metric in a United States Population

Running Title: CCLO Validation in a US Active Surveillance Population

Authors: Joon Yau Leong, BS, ${ }^{1}$ Courtney Capella, BS, ${ }^{1}$ Seth Teplitsky, BS, ${ }^{1}$ Leonard G. Gomella, MD, ${ }^{1}$ Edouard

J. Trabulsi, MD, ${ }^{1}$ Costas D. Lallas, MD, ${ }^{1}$ Thenappan Chandrasekar, MD ${ }^{1}$

Affiliation:

1. Department of Urology, Sidney Kimmel Cancer Center, Thomas Jefferson University, Philadelphia PA, USA

Corresponding Author:

Thenappan Chandrasekar, MD

Department of Urology

1025 Walnut Street, Suite 1112

Philadelphia, PA 19107

United States of America

Phone: 732-742-1025

Fax: 844-351-9508

Email: thenappan.chandrasekar@gmail.com

Funding Source: None

Conflicts of Interest: All authors report no COI.

Article Type/Category: Original

Summary

Manuscript word count (including abstract): 2736

Tables: 3 ( +3 supplementary)

Figures: 3 (+1 supplementary) 


\section{STRUCTURED ABSTRACT}

Background: Treatment progression for men on active surveillance (AS) for prostate cancer (PCa) is driven primarily by grade and volume progression on isolated prostate biopsies (PBX). As PCa is a multifocal disease, regional disease progression over time should be accounted for.

Objective: To validate the utility of the Cumulative Cancer Location (CCLO) metric, which assesses regional core involvement, as described by Erickson et al., in predicting AS outcomes in a North American cohort.

Design, setting, and participants: Single institutional retrospective chart review of all AS patients evaluated between 2015-2017.

Outcome Measurements and Statistical Analysis: CCLO defined as total number of cancerpositive sextant locations among all PBx to that point in time (range 1-6). Baseline demographics and clinical characteristics of the entire cohort were stratified by CCLO $\Delta$, defined as the difference between the first and last CCLO. CCLO $\Delta$ then correlated to progression to treatment and treatment outcomes.

Results: 261 men met inclusion criteria. Though mean number of biopsies was slightly higher in the CCLO $\triangle$ 3-5 cohort than the CCLO $\Delta$ 0-2 cohort ( $p=0.006)$, mean AS follow-up time ( 3.3 years) was not significantly different $(p=0.327)$. As CCLO $\Delta$ increased, the proportion of men remaining on AS decreased while the proportion of men receiving treatment increased $(p<0.001)$. In men undergoing radical prostatectomy, higher CCLO $\Delta$ was not associated with higher rates of Gleason 7-10 $(p=0.38)$ or pT3 $(p=0.52)$ disease. However, as CCLO $\Delta$ increased, upgrading from final PBx to RP pathology increased while downgrading decreased $(p=0.12)$. In Kaplan-Meier analyses, lower CCLO $\triangle$ and lower initial CLO score were associated with the highest 5-year treatment-free survival rates $(p<0.001)$.

Conclusion: Higher regional cancer core involvement is associated with higher rates of progression to treatment in AS patients. The CCLO metric is a potentially useful modality in stratifying patients for treatment in AS patients among the North American cohort, while not compromising disease outcomes.

Patient Summary: In the North American population, cumulative cancer-positive locations among biopsies can be used to predict active surveillance outcomes in men with prostate cancer.

Keywords: active surveillance, prostate cancer, cumulative cancer location 
1

2

3

\section{INTRODUCTION}

Since its introduction, widespread screening with serum prostate-specific antigen (PSA) has facilitated earlier detection of prostate cancer (PCa). ${ }^{1}$ Although the detection rate of PCa has increased, a significant proportion of newly diagnosed PCa are found to be clinically localized low-risk disease. ${ }^{2,3}$ With improved understanding of the indolent natural history of these low risk prostate cancers, active surveillance (AS) has emerged as the standard of care for men with low-risk disease, based on the strength of multiple prospective series that have demonstrated excellent cancer-specific and overall survival without sacrificing an opportunity for cure in men who progress to higher risk disease. ${ }^{4-8}$

While significant variation exists among AS protocols and international guidelines, eligibility criteria for AS typically include a combination of PSA level, PSA density, clinical stage, and prostate biopsy (PBx) data (\% positive cores and core volume) on both diagnostic and confirmatory biopsy. ${ }^{9-12}$ In men followed on AS, progression to intervention is most commonly due to pathologic upgrading or increased tumor volume, but clinicians may also consider PSA kinetics and radiographic upstaging. ${ }^{13,14}$ Treatment progression due to pathologic upgrading, increased tumor volume and fast PSA doubling times are reported in 35-50\%, 2-63\% and 21 $44 \%$ of AS cohorts, respectively. Progression to definitive treatment due to patient anxiety has also been reported at rates of $6-9 \% .^{4-6}$ 
21 Ultimately, the decision to proceed to intervention is driven by the results of the latest PBx,

22 often considered in isolation from prior PBx results. Given the multifocal nature of PCa and the

23 sampling error that accompanies freehand transrectal ultrasonography (TRUS) PBx, ${ }^{15-19}$ Erickson

24 et al. described a novel method that considers the location of positive cores and regional

25 involvement over time. ${ }^{20}$ They first described cumulative cancer locations (CCLO) as a distinct

26 and powerful predictor of AS outcomes (Supplementary Figure 1). Herein, we validate the utility

27 of the CCLO metric in predicting AS outcomes in a North American cohort. 


\section{PATIENTS AND METHODS}

Following institutional review board approval, retrospective chart review was performed on all AS patients evaluated in our institution between 2015-2017. At our institution, we utilize the AUA guidelines for active surveillance in men with very-low risk and low risk patients with localized PCa, and highly selective low volume localized intermediate risk PCa. ${ }^{12}$ Men on active surveillance are followed routinely with PSA testing every 6 months and a PBx every 2 years; PBx may be completed earlier if there is evidence of a rising PSA or abnormal DRE. Patient demographics (age, race, clinical stage, preoperative PSA), clinical outcomes (AS progress, progression to treatment, PCa treatment modality), and radical prostatectomy (RP) pathology synoptic reports were also recorded. Pathology reports of all PBx for individual patients were abstracted for date of procedure, number and location of positive cores, and total Gleason score. Each PBx was reviewed and given a cancer location (CLO) score based on sextant location containing any positive cancer cores as described by Erickson et al. ${ }^{20}$ Cumulative CLO (CCLO) was defined as the sum of all CLOs in all PBx to that point in time, while CCLO $\triangle$ was defined as the difference between the CCLO of the most recent PBx and the CLO of the first PBx (Supplementary Figure 1).

All patients were stratified based on CCLO $\Delta$ scores $(0,1,2,3-5)$, which was then correlated to AS clinical outcomes. Descriptive statistics for demographic and outcome comparisons were performed using analysis of variance (ANOVA) for continuous variables and chi-square test for categorical variables. Kaplan-Meier survival curves were generated to evaluate treatment-free 
49 progression stratified by CCLO $\triangle$ in the entire cohort and sub-stratified by initial CLO; results

50 were compared with the log-rank test. All statistical tests were two-tailed and a p-value of

$51<0.05$ was considered statistically significant. Analyses were completed using SPSS ${ }^{\circledR}$, version

$52 \quad 23.0$. 
53

54

55

56

57

58

59

60 Clinical outcomes

61 Table 2 summarizes clinical outcomes stratified by CCLO $\Delta$. Within the entire cohort, most

62

63

64

65

66

67

68

69

70

71

72

RESULTS

$\underline{\text { Patient demographics }}$

Table 1 highlights key demographic data for the entire cohort and stratified by CCLO $\Delta$. Age, initial PSA, clinical T-stage and risk stratification were not significantly different amongst CCLO $\triangle$ cohorts. Although the mean number of PBx increased with higher CCLO $\Delta(p=0.006)$, the time on active surveillance was not significantly different amongst cohorts $(p=0.327)$.

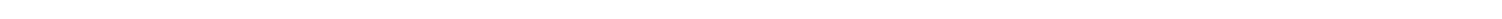
patients remained on AS (55.2\%), while $42.5 \%$ were recommended treatment, with $34.1 \%$ agreeing to undergo treatment. As CCLO $\triangle$ increased, the proportion of men remaining on AS decreased and the proportion of men receiving treatment increased $(p<0.0001)$. Treatment indications and modalities

Table 3 and Supplementary Table 1 summarizes the treatment indications and treatment modalities utilized within each CCLO $\Delta$ cohort, respectively. Across all subsets, the primary indication for treatment recommendation and receipt was pathologic upgrading on PBx, ranging between $70-80 \%$, while increased tumor volume was a much less common indication (20-30\%). Radical prostatectomy (RP) was the most common treatment modality, with $54.7 \%$ of men receiving RP and $45.3 \%$ receiving radiation therapy with or without hormonal therapy. 
Among the 6 (6.3\%) patients who requested treatment due to anxiety, 4 (66.7\%) underwent RP while 2 (33.3\%) underwent radiotherapy.

Analysis of Radical Prostatectomy pathology

Supplementary Table 2 highlights the pathology outcomes in the 52 (19.9\%) men who discontinued AS and underwent RP. Of the 4 patients who voluntarily discontinued AS, 2 had Gleason $3+3$ disease and 2 had Gleason 3+4 disease; all 4 had pT2 disease. A higher CCLO $\Delta$ was not significantly associated with higher rates of intermediate risk (Gleason 7) disease, high risk (Gleason 8-10) disease or non-localized pT3 disease.

\section{Progression of disease}

Figure 1 depicts treatment-free survival (TFS) based on CCLO $\Delta$ for the entire population. Men with CCLO $\Delta 0$ had the best treatment-free survival (5-year TFS 78\%), while men with CCLO $\Delta 1-5$ had a much higher rate of progression to treatment $(5$-year TFS 35-58\%) $(p<0.001)$. Further stratification based on patients initial CLO (Figure 2) demonstrated distinct populations with superior TFS. Men with the best TFS (5-year TFS 90\%) were those with initial CLO 1 and CCLO $\Delta 0$ (Figure 2A).

1 The swimmer's plots in Figure 3 depicts the entire patient cohort stratified by treatment receipt. Figure $3 \mathrm{~A}$ are patients who remained on $\mathrm{AS}$, including men who were recommended 
93 treatment but refused. Figure 3B are patients who received treatment, including those who

94 chose treatment based on personal choice. 


\section{DISCUSSION}

AS has emerged as a standard of care for men with low-risk localized PCa, preserving an opportunity for curative intervention while minimizing overtreatment and associated adverse events. AS is characterized by a $30-40 \%$ rate of progression to treatment, driven primarily by grade and volume progression. ${ }^{21}$ Progression to treatment is typically determined based on a patient's most recent PBx, often in isolation from their prior PBx history. Even when considering volume of disease, clinicians commonly focus on the number and percentage of positive cores within each PBx rather than the cumulative location of positive cores. ${ }^{22}$ In 2018 , Erickson et al. found that regional core involvement from the first two PBx (initial and confirmatory) may represent an additional metric to predicting progression of AS patients to treatment, with higher CCLO scores predicting poorer AS outcomes. ${ }^{20}$ Importantly, the CCLO scores account for regional tumor burden from all prior PBx rather than the most recent PBx alone. As the study by Erickson et al. was conducted in 3 European centers with relatively homogenous populations, herein we independently validate the CCLO metric in a North American cohort. ${ }^{23}$

While previous studies have established that total number of positive PBx cores is predictive of AS progression, Erickson et al. showed that CCLO $\triangle$ was a powerful predictor for AS outcomes. Moreover, their study reports that CCLO $\Delta$ outperformed number of positive cores in predicting AS outcomes, with higher CCLO $\Delta$ predicting shorter treatment free survival on AS, Gleason score upgrading and adverse findings on RP. ${ }^{20}$ In our study, a higher CCLO $\Delta$ was also significantly associated with treatment recommendation and treatment receipt $(p<0.0001)$. 
117 Kaplan-Meier analyses indicate that patients with higher CCLO $\Delta$ have lower 5-year TFS rates.

When stratified by initial volume of disease, it appeared that men with an initial CLO 1 and $\mathrm{CCLO} \Delta 0$ have the greatest benefit from AS, with 5-year TFS rates exceeding $90 \%$. Even men with initial CLO 1 and CCLO $\triangle 1-5$ had 5-year TFS rates of $<65 \%$. These are consistent with findings by Erickson et al., who demonstrated that higher CCLO at the time of confirmatory biopsy predicted significantly shorter TFS when stratified by the number of positive cores. ${ }^{20}$ These results indicate that while initial volume of disease impacts AS outcomes, cumulative volume progression over time must also be accounted for.

While Erickson et al. analyzed only the first two PBx (initial and confirmatory), in our study, we examined all PBx in patients during their entire AS follow-up, enabling better capture of temporal volume progression. ${ }^{20}$ The mean number of PBx in the entire cohort was 3.1, with some patients receiving up to $7 \mathrm{PBx}$ during follow-up. While it would be easy to presume that a patient's CCLO would increase proportionately with time on AS, we found that time on AS was not significantly associated with CCLO $\Delta$. The swimmer's plot (Figure 3 ) clearly illustrates the distinct clinical trajectories of each AS patient over time. Most of the patients who remained on AS (Figure 3A) had low CCLO $\Delta$ scores throughout their surveillance period; many of the men who remained on AS while having high CCLO scores were recommended treatment but refused. In contrast, when looking at the course of men ultimately progressing to treatment (Figure 3B), most of these men had higher CCLO $\Delta$ scores. However, the spread of initial CLO scores is remarkably similar between the groups - indicating that all these men start with low volume 
disease, but a few progresses to higher volume regional disease over time. Yet, as seen by the side by side comparison of Figures $3 \mathrm{~A}$ and $3 \mathrm{~B}$, there are a subset of patients who progress to higher volume disease later in their AS follow-up, demonstrating that cumulative volume progression need not always occur early. This reinforces the need for continued follow-up in all AS patients. These findings further suggest that $\mathrm{CCLO} \Delta$ can be a useful surrogate in predicting outcomes and need for treatment in AS eligible patients in conjunction with other preestablished clinical characteristics.

Within our cohort, $42 \%$ of patients were recommended treatment while $34 \%$ eventually underwent treatment. These rates are consistent with prior literature regarding progression to treatment in the AS population. ${ }^{21}$ In contrast to Erickson et al., who found that higher CCLO was independently associated with adverse RP findings, in our subset of patients who underwent $\mathrm{RP}$, higher CCLO $\Delta$ was not associated with an increased rate of Gleason 7-10 pathology on RP $(p=0.38)$ or non-localized $p$ T3 upstaging $(p=0.52) .{ }^{20}$ Interestingly, we found that as CCLO $\Delta$ increased, there was a suggestion, although not statistically significant, of increased upgrading from final PBx to RP pathology $(p=0.12)$. However, in our cohort, $5.8 \%$ and $36.5 \%$ of patients had Gleason $\geq 8$ disease and pT3 disease, respectively, on final RP pathology, which was consistent with previously reported rates in the literature for Gleason 8-10 upgrading (8.79.2\%) and pT3 upstaging (27.7-43.0\%). ${ }^{24,25}$ Consistent with our data, Dall'Era et al. also found no association between time on AS and adverse pathological outcomes at the time of RP. ${ }^{26}$ Overall, 
the literature supports that men on AS undergoing RP have favorable outcomes, which is likely related to the selective criteria of AS inclusion and the long natural history of low risk PCa.

As for patients who were recommended or received treatment, we found that Gleason upgrading was the most common reason for clinicians to discontinue AS and pursue treatment. In a study of 46 AS patients who subsequently underwent RP, Hong et al. demonstrated that Gleason upgrading from pattern 3 to 4 or 5 was the most common reason for AS discontinuation (45.7\%) and is also the most prognosticating factor for unfavorable disease on RP. Increased tumor volume (21.7\%) and increased percentage of cancer per biopsy core $(8.7 \%)$ were among other common reasons for AS discontinuation. ${ }^{25}$ These findings suggest the negative predictive value of a low CCLO $\triangle$.

Our study is not without its limitations. First, our study design is based on retrospective chart reviews with its inherent limitations. There was no central pathology review of PBx and final RP pathology. Our small sample size may also limit the ability to identify important associations with pathologic outcomes. Having data from a larger number of AS patients would also allow further analysis of patients with higher initial CLO and higher CCLO $\triangle$ and their association with AS outcomes. Additionally, regional core data depended on accurate labeling of PBx cores at the time of biopsy. Lastly, as a tertiary care facility, patient selection may be biased towards higher risk individuals and may not reflect the full spectrum of AS disease pathology. However, 
178 regardless of these limitations, this cohort still represents a moderate AS cohort with a mean 3-

179 year AS follow-up. 
181 CONCLUSION

182 Our findings suggest that regional core involvement of PCa is associated with progression of

183 disease in AS patients. The CCLO metric is a potentially useful modality among the North

184 American cohort for risk stratification in patients managed with AS, without compromising

185 disease outcomes.

186

187 ACKNOWLEDGEMENTS: None 


\section{REFERENCES / BIBLIOGRAPHY}

1. Catalona WJ, Smith DS, Ratliff TL, et al. Measurement of prostate-specific antigen in serum as a screening test for prostate cancer. N Engl J Med. 1991;324(17):1156-1161.

2. Han M, Partin AW, Piantadosi S, Epstein JI, Walsh PC. Era specific biochemical recurrence-free survival following radical prostatectomy for clinically localized prostate cancer. J Urol. 2001;166(2):416-419.

3. Welch HG, Black WC. Overdiagnosis in cancer. J Natl Cancer Inst. 2010;102(9):605-613.

4. Klotz L, Vesprini D, Sethukavalan P, et al. Long-term follow-up of a large active surveillance cohort of patients with prostate cancer. J Clin Oncol. 2015;33(3):272-277.

5. Tosoian JJ, Mamawala M, Epstein JI, et al. Intermediate and Longer-Term Outcomes From a Prospective Active-Surveillance Program for Favorable-Risk Prostate Cancer. J Clin Oncol. 2015;33(30):3379-3385.

6. Bokhorst LP, Valdagni R, Rannikko A, et al. A Decade of Active Surveillance in the PRIAS Study: An Update and Evaluation of the Criteria Used to Recommend a Switch to Active Treatment. Eur Urol. 2016;70(6):954-960.

7. Selvadurai ED, Singhera $M$, Thomas K, et al. Medium-term outcomes of active surveillance for localised prostate cancer. Eur Urol. 2013;64(6):981-987.

8. Welty CJ, Cowan JE, Nguyen $\mathrm{H}$, et al. Extended followup and risk factors for disease reclassification in a large active surveillance cohort for localized prostate cancer. J Urol. 2015;193(3):807-811.

9. Morash C, Tey R, Agbassi C, et al. Active surveillance for the management of localized prostate cancer: Guideline recommendations. Can Urol Assoc J. 2015;9(5-6):171-178.

10. Mohler JL, Armstrong AJ, Bahnson RR, et al. Prostate Cancer, Version 1.2016. J Nat/ Compr Canc Netw. 2016;14(1):19-30.

11. Mottet N, Bellmunt J, Bolla M, et al. EAU-ESTRO-SIOG Guidelines on Prostate Cancer. Part 1: Screening, Diagnosis, and Local Treatment with Curative Intent. Eur Urol. 2017;71(4):618-629.

12. Sanda MG, Cadeddu JA, Kirkby E, et al. Clinically Localized Prostate Cancer: AUA/ASTRO/SUO Guideline. Part II: Recommended Approaches and Details of Specific Care Options. J Urol. 2018;199(4):990-997.

13. Soloway MS, Soloway CT, Williams S, Ayyathurai R, Kava B, Manoharan M. Active surveillance; a reasonable management alternative for patients with prostate cancer: the Miami experience. BJU Int. 2008;101(2):165-169.

14. Bul $M$, Zhu $X$, Valdagni $R$, et al. Active surveillance for low-risk prostate cancer worldwide: the PRIAS study. Eur Urol. 2013;63(4):597-603.

15. Ruijter ET, van de Kaa CA, Schalken JA, Debruyne FM, Ruiter DJ. Histological grade heterogeneity in multifocal prostate cancer. Biological and clinical implications. J Pathol. 1996;180(3):295-299.

16. Häggman M, Nordin B, Mattson S, Busch C. Morphometric studies of intra-prostatic volume relationships in localized prostatic cancer. Br J Urol. 1997;80(4):612-617.

17. Scattoni V, Zlotta A, Montironi R, Schulman C, Rigatti P, Montorsi F. Extended and saturation prostatic biopsy in the diagnosis and characterisation of prostate cancer: a critical analysis of the literature. Eur Urol. 2007;52(5):1309-1322.

18. Lahdensuo K, Mirtti T, Petas A, Rannikko A. Performance of transrectal prostate biopsies in detecting tumours and implications for focal therapy. Scand J Urol. 2015;49(2):90-96.

19. Han M, Chang D, Kim C, et al. Geometric evaluation of systematic transrectal ultrasound guided prostate biopsy. J Urol. 2012;188(6):2404-2409. 
20. Erickson AM, Luzzago S, Semjonow A, et al. Cumulative Cancer Locations is a Novel Metric for Predicting Active Surveillance Outcomes: A Multicenter Study. European Urology Oncology. 2018;1(4):268-275.

21. Komisarenko M, Martin $L$, Finelli A. Active surveillance review: contemporary selection criteria, follow-up, compliance and outcomes. Transl Androl Urol. 2018;7(2):243-255.

22. Amin MB, Lin DW, Gore JL, et al. The critical role of the pathologist in determining eligibility for active surveillance as a management option in patients with prostate cancer: consensus statement with recommendations supported by the College of American Pathologists, International Society of Urological Pathology, Association of Directors of Anatomic and Surgical Pathology, the New Zealand Society of Pathologists, and the Prostate Cancer Foundation. Arch Pathol Lab Med. 2014;138(10):1387-1405.

23. Montinaro F, Busby GB, Pascali VL, Myers S, Hellenthal G, Capelli C. Unravelling the hidden ancestry of American admixed populations. Nat Commun. 2015;6:6596.

24. Imnadze M, Sjoberg DD, Vickers AJ. Adverse Pathologic Features at Radical Prostatectomy: Effect of Preoperative Risk on Oncologic Outcomes. Eur Urol. 2016;69(1):143-148.

25. Hong SK, Sternberg IA, Keren Paz GE, et al. Definitive pathology at radical prostatectomy is commonly favorable in men following initial active surveillance. Eur Urol. 2014;66(2):214-219.

26. Dall'Era MA, Cowan JE, Simko J, et al. Surgical management after active surveillance for low-risk prostate cancer: pathological outcomes compared with men undergoing immediate treatment. BJU Int. 2011;107(8):1232-1237. 
FIGURE LEGENDS

Table 1: Patient demographics

\begin{tabular}{|c|c|c|c|c|c|c|}
\hline & All & CCLO $\Delta 0$ & CCLO $\Delta 1$ & CCLO $\Delta 2$ & CCLO $\Delta 3-5$ & $p$-value \\
\hline Total, N (\%) & $261(100.0)$ & 91 (34.9) & $80(30.7)$ & $62(23.8)$ & $28(10.7)$ & --- \\
\hline Age, years (mean $\pm S D$ ) & $69.5 \pm 7.3$ & $68.7 \pm 7.4$ & $69.6 \pm 8.1$ & $70.5 \pm 6.2$ & $69.5 \pm 7.3$ & 0.551 \\
\hline PSA, ng/mL (mean \pm SD) & $5.3 \pm 2.8$ & $5.2 \pm 2.8$ & $5.4 \pm 2.8$ & $5.5 \pm 2.5$ & $5.2 \pm 3.2$ & 0.904 \\
\hline Number of PBx (mean \pm SD) & $3.1 \pm 1.4$ & $2.8 \pm 1.2$ & $3.1 \pm 1.4$ & $3.2 \pm 1.4$ & $3.8 \pm 1.5$ & 0.006 \\
\hline Time on AS, years (mean \pm SD) & $3.3 \pm 2.5$ & $3.1 \pm 2.5$ & $3.6 \pm 2.6$ & $3.0 \pm 2.4$ & $3.7 \pm 2.6$ & 0.327 \\
\hline Initial CLO (median) & 1 & 1 & 1 & 1.5 & 1 & --- \\
\hline Final CCLO (median) & 2 & 1 & 2 & 3.5 & 4 & --- \\
\hline Gleason Score at $1^{\text {st }}$ PBx & & & & & & 0.111 \\
\hline $3+3, N(\%)$ & $243(93.1)$ & $85(93.4)$ & $76(95.0)$ & $54(87.1)$ & $28(100.0)$ & \\
\hline $3+4, N(\%)$ & $18(6.9)$ & $6(6.6)$. & $4(5.0)$ & $8(12.9)$ & $0(0.0)$ & \\
\hline Clinical T-stage & & & & & & 0.150 \\
\hline cT1, N (\%) & $238(91.2)$ & $84(92.3)$ & $69(86.3)$ & 57 (91.9) & $28(100.0)$ & \\
\hline cT2, N (\%) & $23(8.8)$ & $7(7.7)$ & $11(13.8)$ & $5(8.1)$ & $0(0.0)$ & \\
\hline Risk Stratification & & & & & & 0.669 \\
\hline Very low, N (\%) & 65 (24.9) & $22(24.2)$ & $19(23.8)$ & $15(24.2)$ & $9(32.1)$ & \\
\hline Low, N (\%) & $179(68.6)$ & $62(68.1)$ & $57(71.2)$ & $41(66.1)$ & $19(67.9)$ & \\
\hline Intermediate, N (\%) & $17(6.5)$ & $7(7.7)$ & $4(5.0)$ & $6(9.7)$ & $0(0.0)$ & \\
\hline
\end{tabular}

Abbreviations: PSA - prostate-specific antigen; PBx - prostate biopsy; AS - active surveillance; CLO - cancer location; CCLO cumulative cancer location 
Table 2: Clinical outcomes for AS

\begin{tabular}{|c|c|c|c|c|c|c|}
\hline Clinical Outcomes & All & CCLO $\Delta 0$ & CCLO $\Delta 1$ & CCLO $\Delta 2$ & CCLO $\Delta 3-5$ & $p$-value \\
\hline Total, N (\%) & $261(100.0)$ & $91(100.0)$ & $80(100.0)$ & $62(100.0)$ & $28(100.0)$ & --- \\
\hline Remained on AS, N (\%) & $144(55.2)$ & $71(78.0)$ & $46(57.5)$ & $19(30.6)$ & $8(28.6)$ & \multirow{4}{*}{$<0.0001$} \\
\hline Treatment recommended, N (\%) & $22(8.4)$ & $7(7.7)$ & $4(5.0)$ & $6(9.7)$ & 5 (17.9) & \\
\hline Treatment received, $\mathrm{N}(\%)$ & $89(34.1)$ & $12(13.2)$ & $29(36.3)$ & $33(53.2)$ & $15(53.6)$ & \\
\hline Treatment requested, N (\%) & $6(2.3)$ & $1(1.1)$ & $1(1.3)$ & $4(6.5)$ & $0(0.0)$ & \\
\hline
\end{tabular}

Legend:

Treatment recommended: patients who were recommended treatment but chose to remain on AS

Treatment requested: patients who voluntarily opted out of AS to undergo definitive treatment

Treatment received: patients for whom treatment was recommended and received 
Table 3: Treatment indications

\begin{tabular}{|c|c|c|c|c|c|c|}
\hline & Indication for treatment & All & $\mathrm{CCLO} \Delta 0$ & CCLO $\Delta 1$ & $\mathrm{CCLO} \Delta 2$ & CCLO $\Delta 3-5$ \\
\hline \multirow{4}{*}{$\begin{array}{l}\text { Treatment } \\
\text { recommended, } \\
\text { N (\%) }\end{array}$} & Total & $22(100.0)$ & $7(100.0)$ & $4(100.0)$ & $6(100.0)$ & $5(100.0)$ \\
\hline & Gleason upgrading & $15(68.2)$ & $5(71.4)$ & $3(75.0)$ & $3(50.0)$ & $4(80.0)$ \\
\hline & Increased tumor volume & $9(40.9)$ & $2(28.6)$ & $1(25.0)$ & $3(50.0)$ & $3(60.0)$ \\
\hline & Elevated PSA & $2(9.1)$ & $1(14.3)$ & $0(0.0)$ & $0(0.0)$ & $1(20.0)$ \\
\hline \multirow{4}{*}{$\begin{array}{l}\text { Treatment } \\
\text { received, } \\
\text { N (\%) }\end{array}$} & Total & 89 (100.0) & $12(100.0)$ & $29(100.0)$ & $33(100.0)$ & 15 (100.0) \\
\hline & Gleason upgrading & $72(80.9)$ & $9(75.0)$ & $26(89.7)$ & $25(75.8)$ & $12(80.0)$ \\
\hline & Increased tumor volume & $21(23.6)$ & $2(16.7)$ & $5(17.2)$ & $10(30.3)$ & $4(26.7)$ \\
\hline & Elevated PSA & $7(7.9)$ & $1(8.3)$ & $3(10.3)$ & $1(3.0)$ & $2(13.3)$ \\
\hline
\end{tabular}

*Treatment indications are not mutually exclusive 

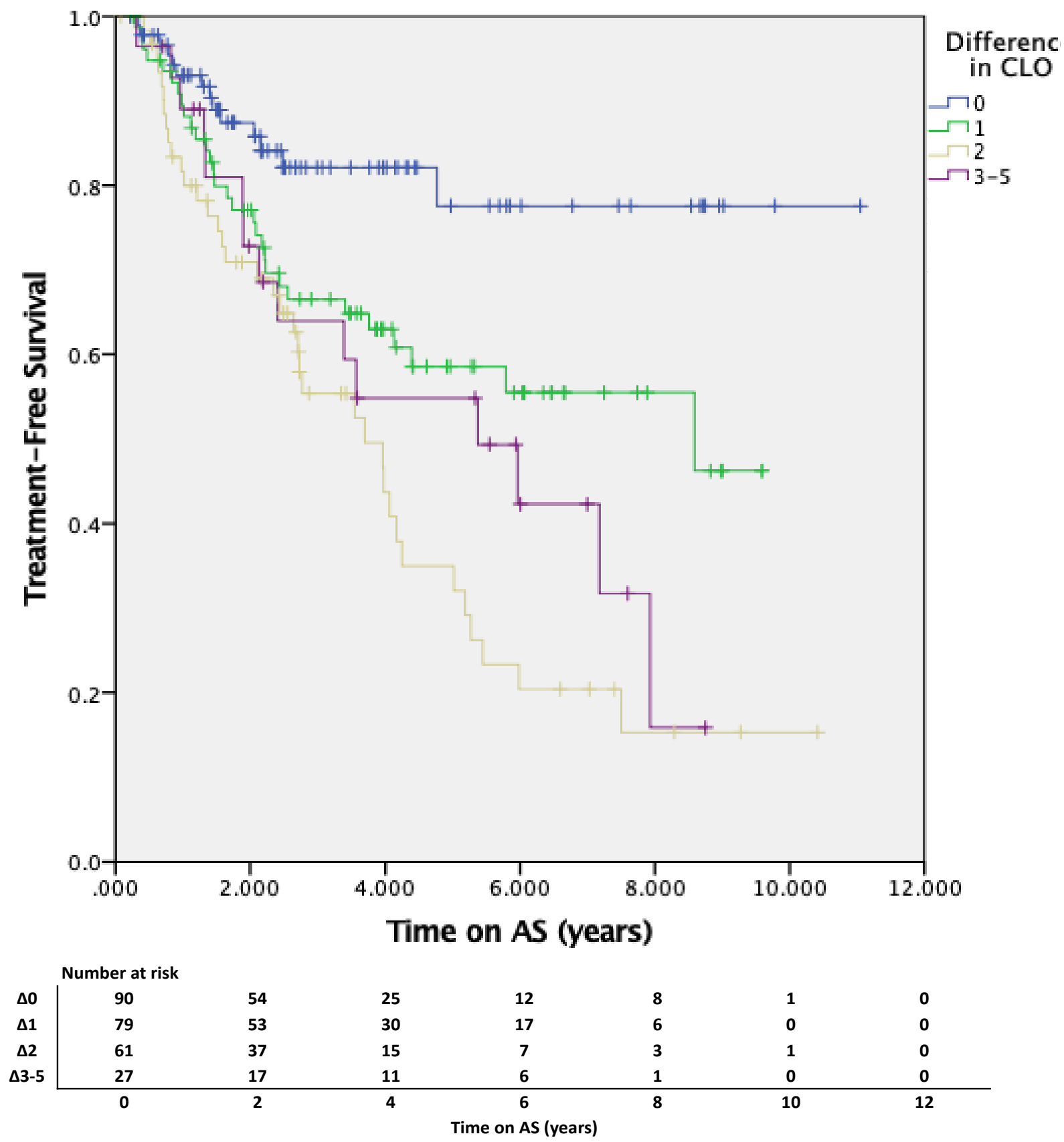

\begin{tabular}{|c|c|c|c|c|c|c|c|}
\hline \multicolumn{8}{|c|}{ Cumulative number of events } \\
\hline$\Delta \mathbf{0}$ & 0 & 10 & 13 & 14 & 14 & 14 & 14 \\
\hline$\Delta \mathbf{1}$ & 0 & 17 & 26 & 29 & 29 & 30 & 30 \\
\hline$\Delta 2$ & 0 & 17 & 28 & 36 & 37 & 37 & 37 \\
\hline \multirow[t]{2}{*}{$\Delta 3-5$} & 0 & 7 & 11 & 13 & 15 & 15 & 15 \\
\hline & 0 & 2 & 4 & 6 & 8 & 10 & 12 \\
\hline
\end{tabular}

Figure 1: Treatment-free survival for the entire population, stratified by CCLO $\Delta$; Log-rank test: $p<0.001$. 

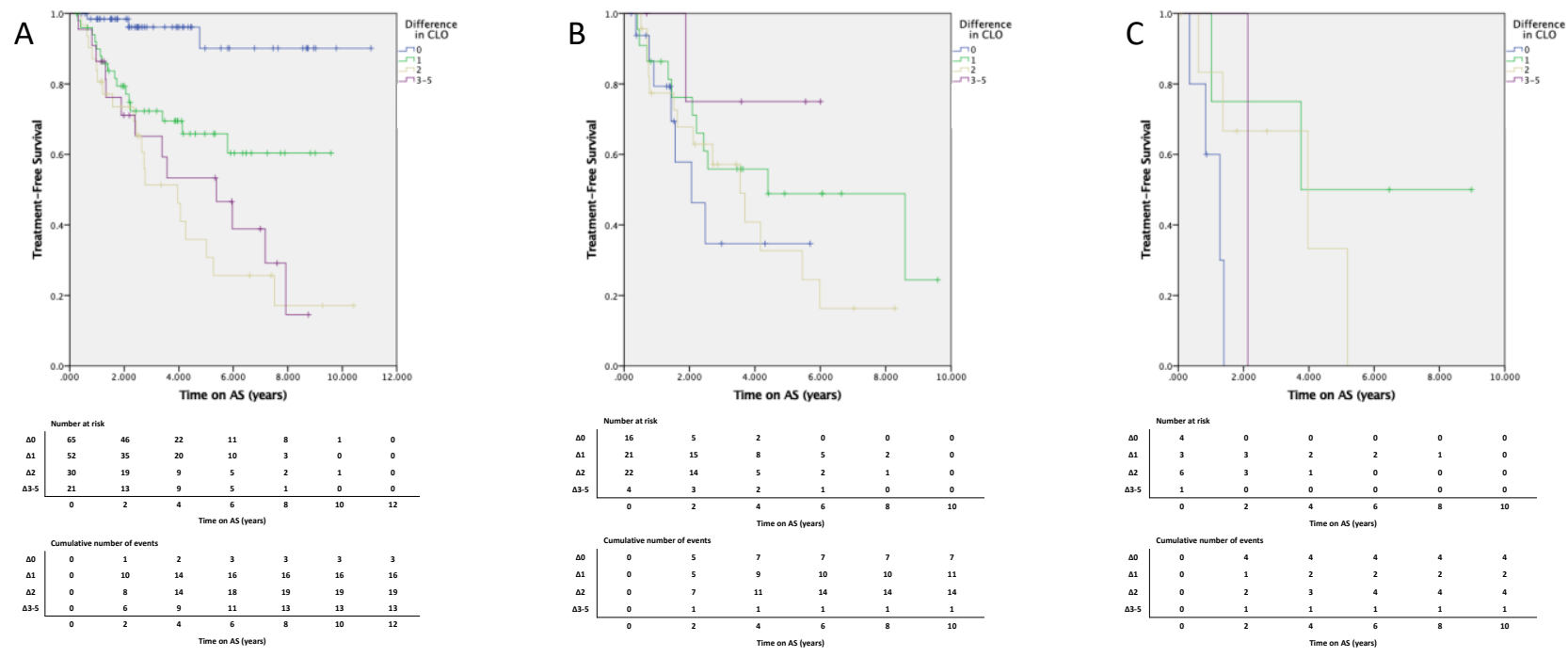

Figure 2: Treatment-free survival stratified by CCLO $\Delta$; Subset analysis of men with initial CLO 1 (Figure 2A), initial CLO 2 (Figure 2B), initial CLO 3 (Figure 2C). 

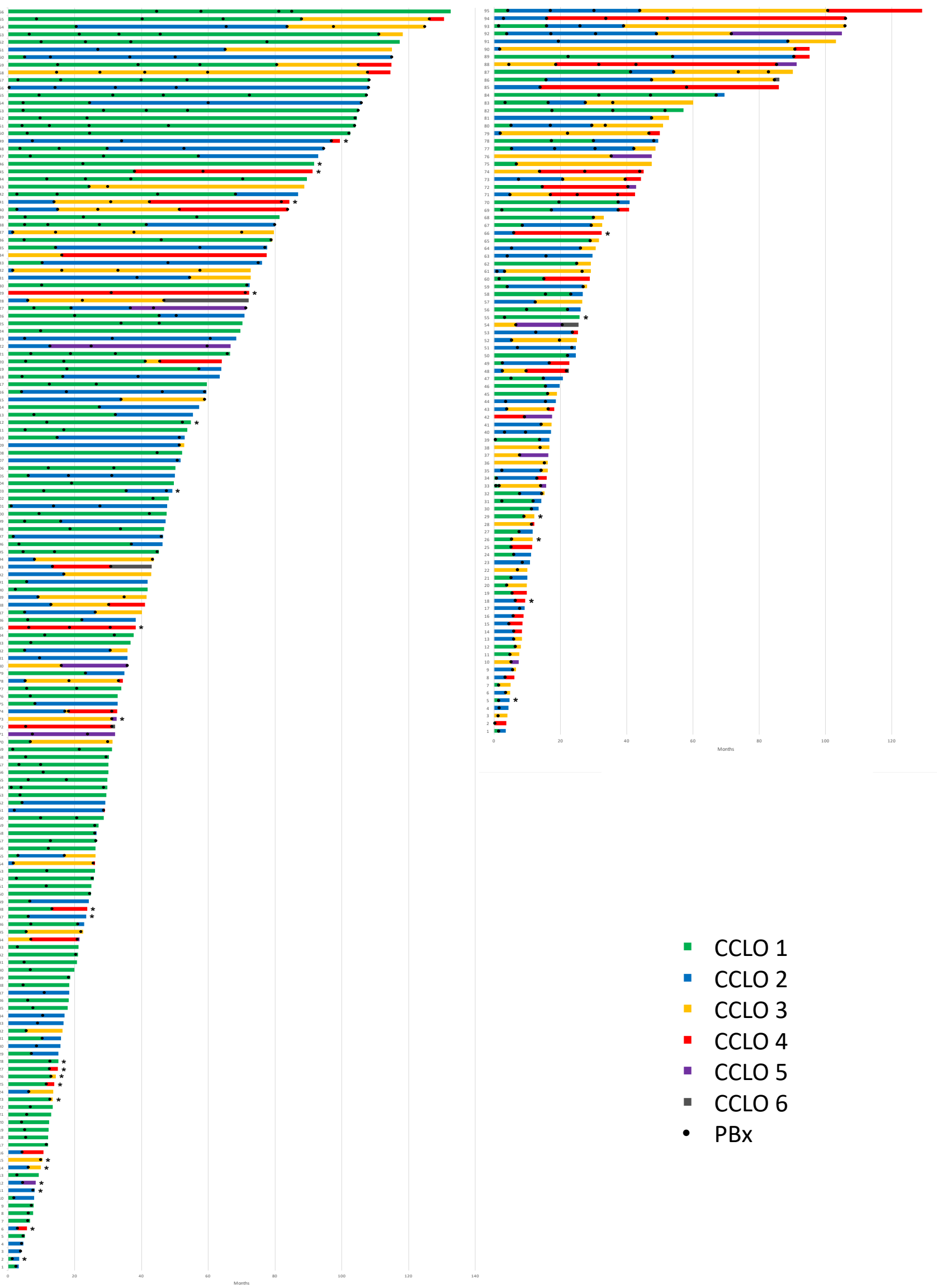
Figure 3: Swimmer's Plots of the Entire Cohort, separated in men who stayed on AS (Figure 3A) and men who received treatment (Figure 3B).

Legend:

Each $\bullet$ represents a single biopsy. Color coding represents the CCLO at the time based on all prior biopsies.

In Figure 3A, * represents men recommended for treatment but who refused.

In Figure 3B, * represents men who chose treatment as a personal choice. 


\section{SUPPLEMENTARY TABLES}

Supplementary Table 1: Treatment modalities

\begin{tabular}{|l|l|l|l|l|l|}
\hline Types of Treatment & All & CCLO $\Delta \mathbf{0}$ & CCLO $\Delta \mathbf{1}$ & CCLO $\Delta \mathbf{2}$ & CCLO $\Delta \geq \mathbf{3}$ \\
\hline Total, N (\%) & $95(100.0)$ & $13(100.0)$ & $30(100.0)$ & $37(100.0)$ & $15(100.0)$ \\
\hline RP, N (\%) & $52(54.7)$ & $10(76.9)$ & $19(63.3)$ & $15(40.5)$ & $8(53.3)$ \\
\hline XRT +/- ADT, N (\%) & $43(45.3)$ & $3(23.1)$ & $11(36.7)$ & $22(59.5)$ & $7(46.7)$ \\
\hline
\end{tabular}

Abbreviations: RP - radical prostatectomy; XRT - radiation therapy; ADT - hormonal therapy; CCLO - cumulative cancer location.

*Treatment modalities are not mutually exclusive

Supplementary Table 2: Analysis of RP patients

\begin{tabular}{|c|c|c|c|c|c|c|c|}
\hline \multicolumn{2}{|l|}{ RP outcomes } & All & $\mathrm{CCLO} \Delta 0$ & CCLO $\Delta 1$ & CCLO $\Delta 2$ & $\operatorname{CCLO} \Delta \geq 3$ & $p$-value \\
\hline \multicolumn{2}{|l|}{ Total } & $52(100.0)$ & $10(100.0)$ & $18(100.0)$ & $15(100.0)$ & $9(100.0)$ & --- \\
\hline \multirow{4}{*}{$\begin{array}{l}\text { Gleason score, } \\
\mathrm{N}(\%)\end{array}$} & $3+3$ & 9 (17.3) & $3(30.0)$ & $2(11.1)$ & $2(13.3)$ & $2(22.2)$ & \multirow{4}{*}{0.380} \\
\hline & $3+4$ & $31(59.6)$ & $5(50.0)$ & $9(50.0)$ & $12(80.0)$ & $5(55.5)$ & \\
\hline & $4+3$ & $9(17.3)$ & $1(10.0)$ & $6(33.3)$ & $0(0.0)$ & $2(22.2)$ & \\
\hline & $8-10$ & $3(5.8)$ & $1(10.0)$ & $1(5.6)$ & $1(6.7)$ & $0(0.0)$ & \\
\hline \multirow{2}{*}{$\begin{array}{l}\text { Pathological T- } \\
\text { stage, N (\%) }\end{array}$} & pT2 & $33(63.5)$ & $7(70.0)$ & $9(50.0)$ & $11(73.3)$ & $6(66.7)$ & \multirow{2}{*}{0.520} \\
\hline & pT3 & $19(36.5)$ & $3(30.0)$ & $9(50.0)$ & $4(26.7)$ & $3(33.3)$ & \\
\hline
\end{tabular}

Abbreviations: RP - radical prostatectomy; CCLO - cumulative cancer location

Supplementary Table 3: Gleason Score comparison of final PBx to RP

\begin{tabular}{|l|l|l|l|l|l|l|}
\hline RP outcomes & All & CCLO $\Delta \mathbf{0}$ & CCLO $\Delta \mathbf{1}$ & CCLO $\Delta \mathbf{2}$ & CCLO $\Delta \geq 3$ & $p$-value \\
\hline Total, N (\%) & $52(100.0)$ & $10(100.0)$ & $18(100.0)$ & $15(100.0)$ & $9(100.0)$ & --- \\
\hline Pathology downgrade, N (\%) & $7(13.5)$ & $3(30.0)$ & $4(22.2)$ & $0(0.0)$ & $0(0.0)$ & \\
\cline { 1 - 5 } & & & & & & \\
\hline Pathology consistent, N (\%) & $35(67.3)$ & $7(70.0)$ & $11(61.1)$ & $10(66.7)$ & $7(77.8)$ & \\
\hline Pathology upgrade, N (\%) & $10(19.2)$ & $0(0.0)$ & $3(16.7)$ & $5(33.3)$ & $2(22.2)$ & \\
\hline
\end{tabular}

Abbreviations: PBx - prostate biopsy; RP - radical prostatectomy; CCLO - cumulative cancer location 


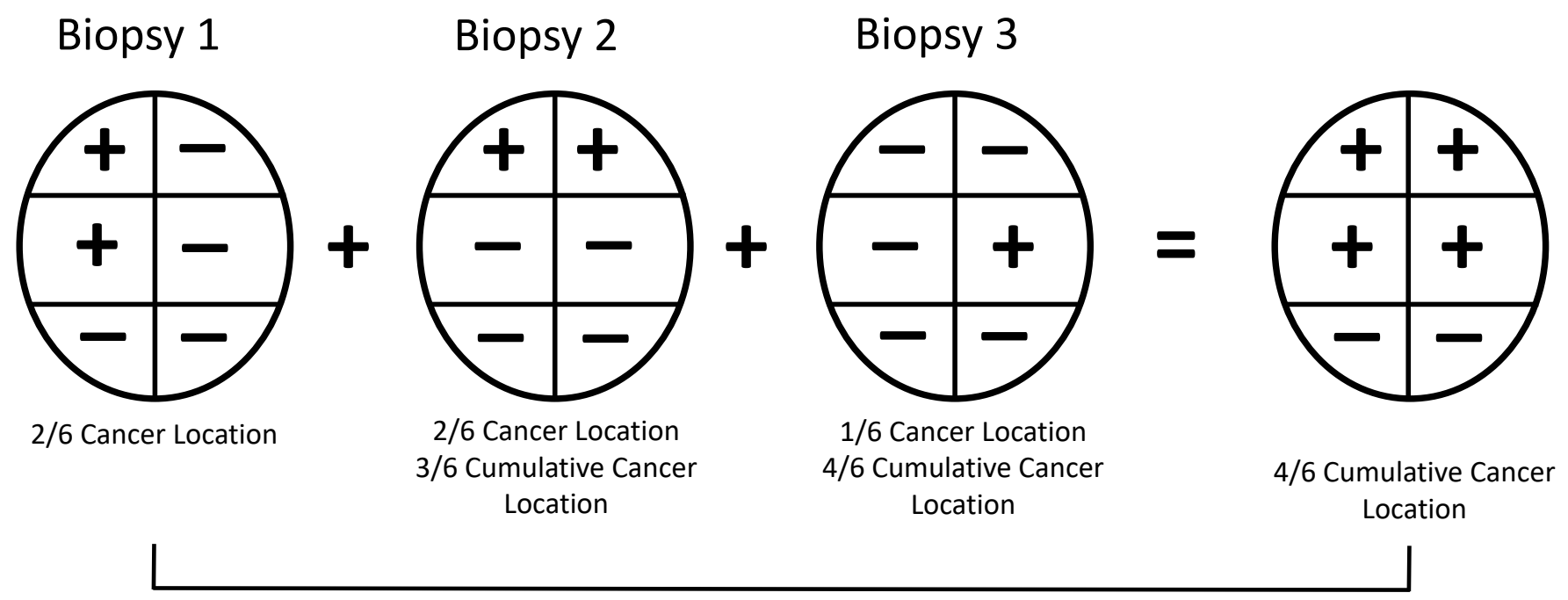

Difference between Cumulative Cancer Locations $(C C L O \Delta)=2$

- CLO - Cancer Location

- CCLO - Cumulative Cancer Location

Supplementary Figure 1: A sample patient on active surveillance for prostate cancer with three prior prostate biopsies. Based on individual biopsies, the patient only has up to 2 cancer-positive locations (CLO). After aggregating CLOs among all prior biopsies, cumulative cancer-positive location (CCLO) is 4 . The CCLO $\triangle$ in this patient, defined by subtracting final CCLO with initial CLO, is 2. 